\title{
Editorial
}

\section{Natural Circulation in Nuclear Reactor Systems}

\author{
Dilip Saha' ${ }^{1}$ and John Cleveland ${ }^{2}$ \\ ${ }^{1}$ Reactor Engineering Division, Bhabha Atomic Research Centre, Trombay, Mumbai 400085, India \\ ${ }^{2}$ Nuclear Power Technology Development Section, Division of Nuclear Power, International Atomic Energy Agency, \\ Wagramer Strasse 5, P. O. Box 100, 1400 Vienna, Austria \\ Correspondence should be addressed to Dilip Saha, dsaha@barc.gov.in
}

Received 2 April 2008; Accepted 2 April 2008

Copyright (C) 2008 D. Saha and J. Cleveland. This is an open access article distributed under the Creative Commons Attribution License, which permits unrestricted use, distribution, and reproduction in any medium, provided the original work is properly cited.

It gives us great pleasure to bring out this special issue on "Natural circulation in nuclear reactor systems" which assumes special significance in the context of present energy technology scenarios. Today nuclear energy produces about $15 \%$ of total world electricity. However, public concern about the safety of nuclear plants has resulted in sociopolitical constraints on its use in some countries. Now a worldwide renewed interest in nuclear energy is evident which is caused mainly by the following factors: (a) progressively dwindling world reserve of fossil fuel, (b) a deep-rooted concern about global warming, (c) increasing oil price, and (d) good performance of current plants. These factors are leading to rising expectations for nuclear energy for the future.

For the sustenance of this renewed interest, besides fuel resource, a number of important issues are being addressed leading to the development of advanced reactor designs as well as fuel cycle technologies. The major issues, which these advanced reactors and fuel cycle concepts are addressing, include economic competitiveness, achieving very high level of safety, waste disposal, environmental effects and proliferation resistance.

An important feature of several advanced reactors designs is the incorporation of passive safety systems. The IAEA conference on "The Safety of Nuclear Power: Strategy for the Future," convened in 1991, recommended that for new plants "the use of passive safety features is a desirable method of achieving simplification and increasing the reliability of the performance of essential safety functions and should be used wherever appropriate." Nuclear plant designers select active safety systems, passive safety systems, or combinations considering fulfilment of required safety functions with sufficient reliability, and the impact on plant operation and cost. A number of passive systems incorporated in advanced reactors employ natural circulation as the mode of energy removal underlining the importance of natural circulation in nuclear reactor design

By definition, natural circulation is a process in which the fluid motion is driven by a density gradient and no external source of energy is required. However, the driving head for natural circulation is low and can be influenced by small changes in operating conditions. Sometimes the flow is not fully developed and can be multidimensional in nature. All these have led to the need of thoroughly understanding the phenomena involved to ensure reliability of natural circulation systems. This has necessitated dissemination of knowledge in this complex and important area. This special issue is a timely and very effective step in this direction.

The papers in this issue cover most of the important aspects of natural circulation-modeling and code development, experimental investigations, development of performance evaluation tools, flow instabilities, safety analysis, and lastly reliability of natural circulation systems.

This issue has been a modest effort to bring to the readers an update on a subject of importance to the reactor designers. We are sure that the readers of this issue will find the papers of immense value and get provoked to explore further in this area.

Dilip Saha John Cleveland 

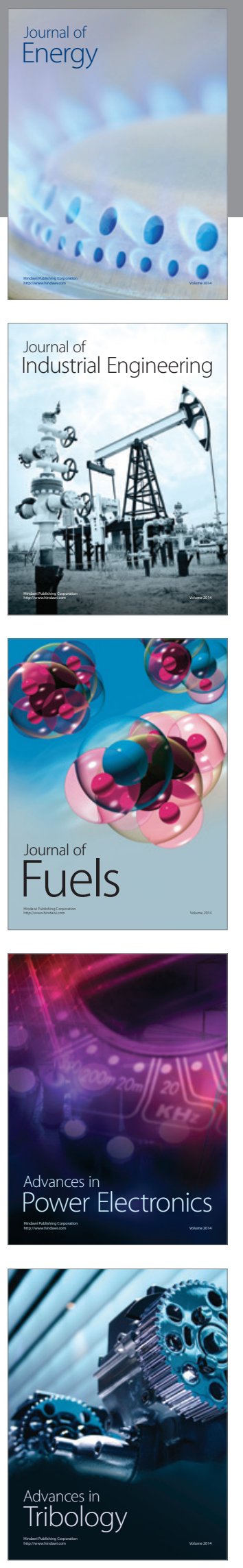
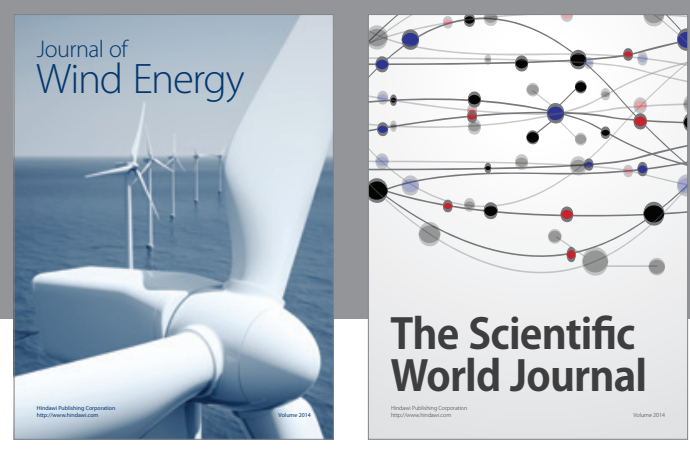

The Scientific World Journal

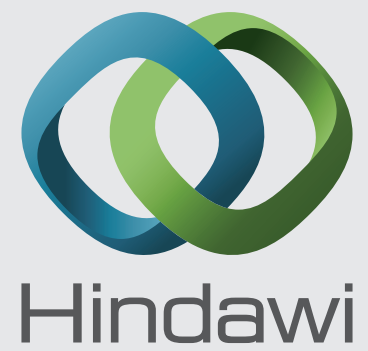

Submit your manuscripts at http://www.hindawi.com
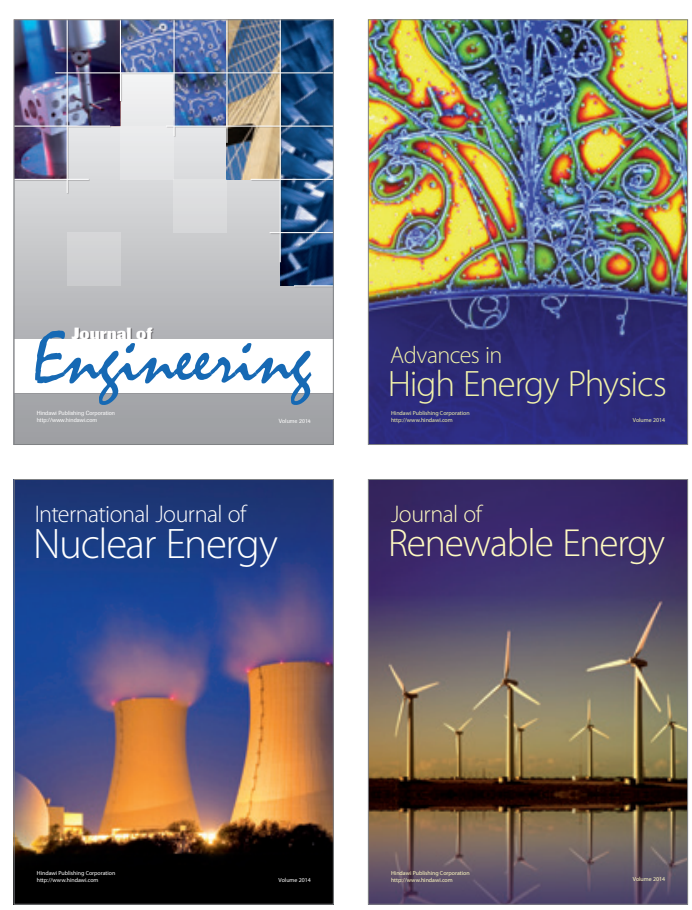

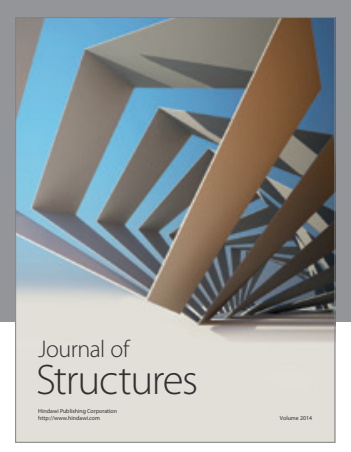

Rotating
Mechinery
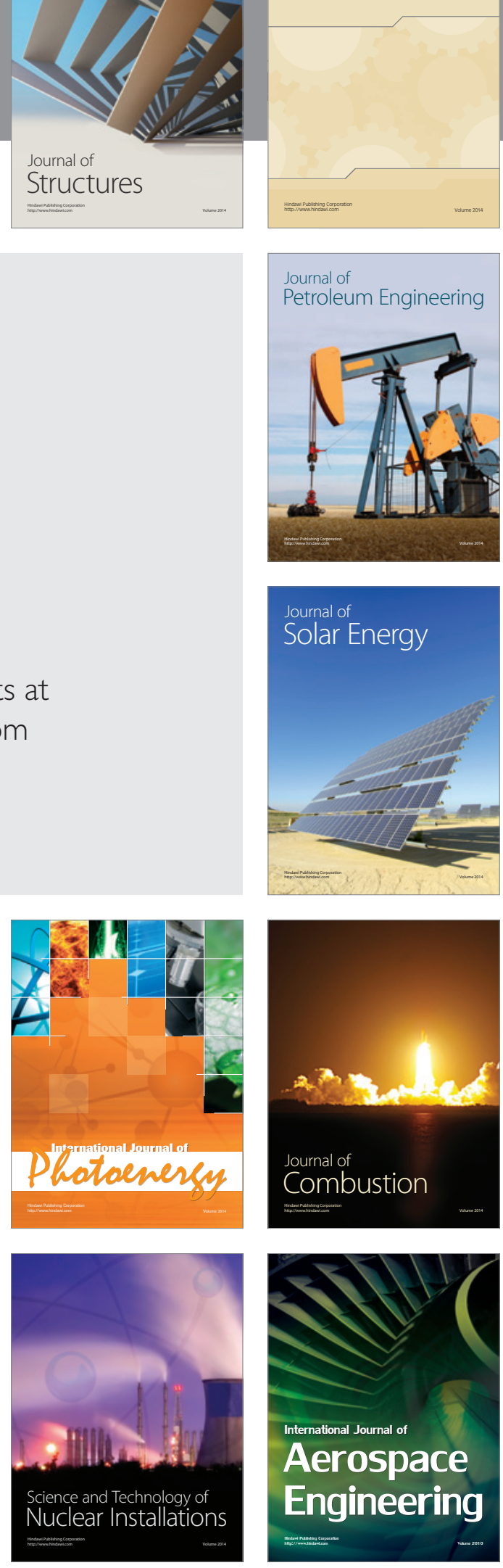